\title{
Bounding formulae for capacitance of cylindrical capacitor with non-homogeneous dielectric material
}

\author{
ISTVÁN ECSEDI ${ }^{1}$, ÁKOS JÓZSEF LENGYEL ${ }^{2 *}$ \\ ${ }^{1,2}$ Institute of Applied Mechanics \\ ${ }^{1,2}$ University of Miskolc \\ ${ }^{1,2} \mathrm{H}-3515$, Miskolc-Egyetemváros, Miskolc \\ ${ }^{1,2}$ HUNGARY
}

\begin{abstract}
The paper deals with the capacitance of cylindrical capacitor which consists of non-homogeneous dielectric materials. The infinite long cylindrical surfaces of capacitor have uniform distributed electric charges in axial direction, so that electrostatic boundary value problem which determines the electric field in the nonhomogeneous dielectric material is a two-dimensional boundary-value problem. The derivation of the bounding formulae is based on the Cauchy-Schwarz inequality. Examples illustrate the applications of the derived upper and lower bound formulae.
\end{abstract}

Key-Words: capacitor, capacitance, electrostatic, cylindrical, upper and lower bounds

Received: March 3, 2021. Revised: September 5, 2021. Accepted: September 30, 2021. Published: October 27, 2021.

\section{Introduction}

The paper deals with the capacitance of a cylindrical capacitor whose generators are infinitely long. The cylindrical capacitor consists of non-homogeneous dielectric materials and it has uniformly distributed electric charges in axial direction on its cylindrical boundary surfaces so that the electrostatic boundaryvalue problem what needs to be solved in order to get the capacitance is a two-dimensional boundary-value problem. The presented bounding formulae give the bounds for capacitance of unit length of cylindrical capacitor. To obtain upper and lower bounds for the unit length of cylindrical capacitance the CauchySchwarz inequality relation and the equations of static electric field are used.

The capacitance is the ability of a capacitor to store electric charge per unit voltage across its inner and outer conductor surfaces. The capacitance is a function depending only on the geometry of capacitor and the permittivity of the dielectric materials between the conductor surfaces of the capacitor. The exact (strict) value of the capacitance is known only for capacitors with very simple shapes, therefore, the principles and the methods that can be used for creating lower and upper bounds to the numerical value of capacitance are important. The derivation of the upper and lower bounds for the capacitance of cylindrical capacitor with non-homogeneous dielectric materials is based on the Cauchy-Schwarz inequality.

Bai and Lonngren [6] solved the spherical capacitor at first analytically and then the obtained solution was compared with an easily determined numerical solution. The presented numerical solution is based on the division of the spherical surface of capacitor into a very large number of subareas. In paper by
Bartlett and Corle the potential distribution for parallel plate capacitor was calculated using both the Love integral equation method and the relaxation method [7]. These two methods give the same result. A system of integral equations for capacitor composed of two discs of different radii is used to get the capacitance in [8]. The complete asymptotic form of the capacitance matrix for large and small distances was derived by Paffuti et al. [8]. Parr [9] dealt with the determination of capacitance of the regular solids. The author provided upper and lower bounds for the Dirichlet integral in order to get the numerical bounds for the capacitance of the five regular solids [9]. In [10] Kammler gave error bounds for the elements of capacitance matrix for a system of conductors. The approximate solution which is used to derive error bound satisfies the field equations of electricity and only almost satisfies the corresponding boundary conditions [10]. Wintle calculated improved upper and lower bounds for the capacitance of a cube [11]. The Kelvin inversion was combined with a random walk method in [11]. A solution of Love's integral equation which forms the base for the analysis of electrostatic field due to equal circular coaxial parallel plates is considered in paper [12]. An explicit analytical solution is obtained for the capacitance when the ratio of distance of plates to radius of plates is greater than two [12]. Sloggett et al. developed a highly accurate numerical model to get the electric field between parallel disc electrodes [13]. Simple analytical expression for the fringing capacitance of parallel plate electrode system of arbitrary shape is given in [13].

Paper by Nithin and Kittur [14] present a design of spiral inductor for millimeter wave oscillator. A set area limits for the optimum dimensions of spiral 

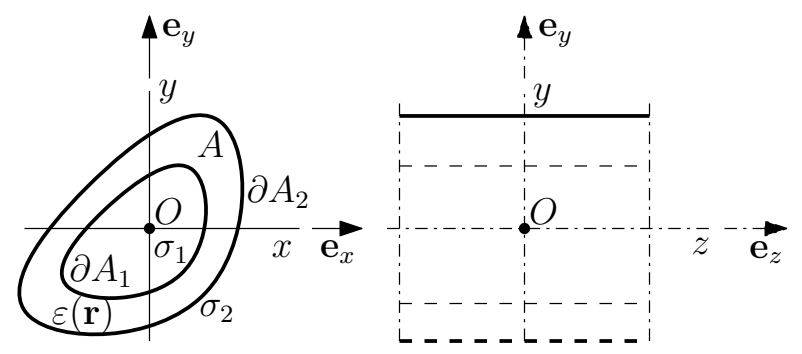

Figure 1: Hollow cylindrical body.

inductor is given in [14]. Arada [15] used a computer simulation method to estimate how varying the dipol electrical length affect to the performance of direction of arrival estimation under the mutual coupling in uniform linear array of the dipol element.

\section{Governing equations of}

\section{two-dimensional electrostatic field}

Fig. 11 shows a two-dimensional domain $A$ whose inner boundary curve is $\partial A_{1}$ and outer boundary curve is $\partial A_{2}$. The boundary curves $\partial A_{1}$ and $\partial A_{2}$ are conductors with surface electric charges $\sigma_{1}=\sigma_{1}\left(s_{1}\right)$, $\sigma_{2}=\sigma_{2}\left(s_{2}\right)$ where $s_{1}$ and $s_{2}$ are arc-lengths defined on the boundary curves $\partial A_{1}$ and $\partial A_{2}$, respectively. Since static electric problems are considered we have

$$
\oint_{\partial A_{1}} \sigma_{1}\left(s_{1}\right) \mathrm{d} s_{1}+\oint_{\partial A_{2}} \sigma_{2}\left(s_{2}\right) \mathrm{d} s_{2}=0 .
$$

The permittivity of the dielectric material between the closed curves $\partial A_{1}$ and $\partial A_{2}$ depends on the position vector $\mathbf{r}=x \mathbf{e}_{x}+y \mathbf{e}_{y}$. Here, $x, y$ are Cartesian coordinates of the $O x y z$ Cartesian coordinate system where $z$ is the axial coordinate and $\mathbf{e}_{x}, \mathbf{e}_{y}, \mathbf{e}_{z}$ are the unit vectors in $x, y$ and $z$ directions (Fig. 1). To give the concept of capacitor we define the following boundaryvalue problem for the two-dimensional doubly connected plane domain $\bar{A}=A \cup \partial A_{1} \cup \partial A_{2}$

$$
\begin{gathered}
\nabla \cdot(\varepsilon(\mathbf{r}) \nabla U)=0, \quad \mathbf{r} \in A, \\
U=0, \quad \mathbf{r} \in \partial A_{2}, \\
U=U_{1} \neq 0, \quad \mathbf{r} \in \partial A_{1} .
\end{gathered}
$$

In Eqs. (2), (3) $U=U(\mathbf{r})$ is the electric potential, $\nabla$ is the two-dimensional del operator [1, 2, 3, 5]. In Cartesian coordinates

$$
\nabla=\frac{\partial}{\partial x} \mathbf{e}_{x}+\frac{\partial}{\partial y} \mathbf{e}_{y}
$$

and in cylindrical coordinates

$$
\begin{gathered}
r=\sqrt{x^{2}+y^{2}}, \quad \varphi=\arctan \frac{y}{x} \\
\nabla=\frac{\partial}{\partial r} \mathbf{e}_{r}+\frac{1}{r} \frac{\partial}{\partial \varphi} \mathbf{e}_{\varphi},
\end{gathered}
$$

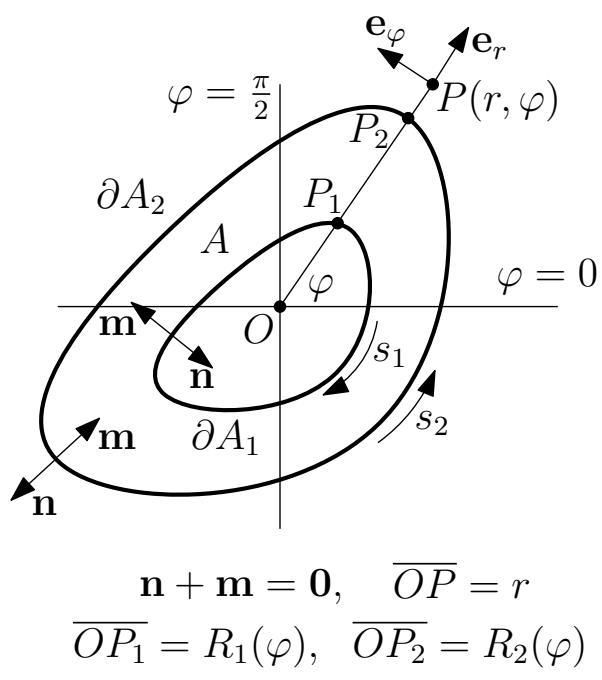

Figure 2: Cylindrical coordinates.

where $\mathbf{e}_{r}(\varphi)$ and $\mathbf{e}_{\varphi}(\varphi)$ are the unit vectors of the cylindrical coordinate system $\operatorname{Or} \varphi$ (Fig. 2). The dot between two vectors denotes their scalar (inner) product and $\varepsilon$ is the dielectric constant. Eq. (2) in detailed form is as follows

$$
\varepsilon(\mathbf{r}) \triangle U+\nabla \varepsilon \cdot \nabla U=0, \quad \mathbf{r} \in A
$$

Here

$$
\begin{gathered}
\triangle=\nabla \cdot \nabla=\frac{\partial^{2}}{\partial x^{2}}+\frac{\partial^{2}}{\partial y^{2}}= \\
=\frac{\partial^{2}}{\partial r^{2}}+\frac{1}{r} \frac{\partial}{\partial r}+\frac{1}{r^{2}} \frac{\partial^{2}}{\partial \varphi^{2}}
\end{gathered}
$$

is the Laplace's operator. We introduce a new function which is defined as

$$
U(\mathbf{r})=U_{1} u(\mathbf{r}), \quad \mathbf{r} \in A \cup \partial A .
$$

It is evident that $u=u(\mathbf{r})$ is the solution of the following boundary-value problem [1, 2, 3, 4, 5]

$$
\begin{gathered}
\nabla \cdot(\varepsilon(\mathbf{r}) \nabla u)=0, \quad \mathbf{r} \in A, \\
u(\mathbf{r})=1, \quad \mathbf{r} \in \partial A_{1}, \\
u(\mathbf{r})=0, \quad \mathbf{r} \in \partial A_{2} .
\end{gathered}
$$

Here we note that $u=u(\mathbf{r})$ is unit free. The governing equations of static electric fields which are used $[1,2,3,4,5]$

$$
\begin{gathered}
\mathbf{E}=-\nabla U=-U_{1} \nabla u, \\
\mathbf{D}=\varepsilon(\mathbf{r}) \mathbf{E}=-U_{1} \varepsilon(\mathbf{r}) \nabla u,
\end{gathered}
$$

$$
\sigma_{1}=\mathbf{D} \cdot \mathbf{m}=-U_{1} \varepsilon(\mathbf{r}) \mathbf{m} \cdot \nabla u, \quad \mathbf{r} \in \partial A_{1},
$$




$$
\sigma_{2}=\mathbf{D} \cdot \mathbf{m}=-U_{1} \varepsilon(\mathbf{r}) \mathbf{m} \cdot \nabla u, \quad \mathbf{r} \in \partial A_{2},
$$

where $\mathbf{m}$ is the inner normal vector of boundary curve $\partial A=\partial A_{1} \cup \partial A_{2}$ (Fig. 2). The whole charge on the conductor boundary curves $\partial A_{1}$ and $\partial A_{2}$ are (Fig. 2)

$$
\begin{gathered}
Q_{1}=-U_{1} \oint_{\partial A_{1}} \varepsilon(\mathbf{r}) \mathbf{m} \cdot \nabla u \mathrm{~d} s_{1}= \\
=U_{1} \oint_{\partial A_{1}} \varepsilon(\mathbf{r}) \mathbf{n} \cdot \nabla u \mathrm{~d} s_{1}= \\
=U_{1} \oint_{\partial A_{1}} \varepsilon(\mathbf{r}) \frac{\partial u}{\partial n} \mathrm{~d} s, \quad \mathbf{n}=-\mathbf{m}, \\
Q_{2}=-U_{1} \oint_{\partial A_{2}} \varepsilon(\mathbf{r}) \mathbf{m} \cdot \nabla u \mathrm{~d} s_{2}= \\
=U_{1} \oint_{\partial A_{2}} \varepsilon(\mathbf{r}) \mathbf{n} \cdot \nabla u \mathrm{~d} s_{2}= \\
=U_{1} \oint_{\partial A_{2}} \varepsilon(\mathbf{r}) \frac{\partial u}{\partial n} \mathrm{~d} s, \quad \mathbf{n}=-\mathbf{m} .
\end{gathered}
$$

It is very easy to show that

$$
Q_{1}+Q_{2}=0,
$$

since

$$
\begin{aligned}
& \int_{A} \nabla \cdot(\varepsilon(\mathbf{r}) \nabla u) \mathrm{d} A=\oint_{\partial A} \varepsilon(\mathbf{r}) \frac{\partial u}{\partial n} \mathrm{~d} s= \\
= & \oint_{\partial A_{1}} \varepsilon(\mathbf{r}) \frac{\partial u}{\partial n} \mathrm{~d} s_{1}+\oint_{\partial A_{2}} \varepsilon(\mathbf{r}) \frac{\partial u}{\partial n} \mathrm{~d} s_{2}=0 .
\end{aligned}
$$

From Eq. (9) it follows that

$$
\begin{gathered}
\int_{A} u \nabla \cdot(\varepsilon(\mathbf{r}) \cdot \nabla u) \mathrm{d} A= \\
=\oint_{\partial A_{1}} \varepsilon(\mathbf{r}) \frac{\partial u}{\partial n} \mathrm{~d} s_{1}-\int_{A} \varepsilon(\mathbf{r})|\nabla u|^{2} \mathrm{~d} A=0
\end{gathered}
$$

that is

$$
\int_{A} \varepsilon(\mathbf{r})|\nabla u|^{2} \mathrm{~d} A=\oint_{\partial A_{1}} \varepsilon(\mathbf{r}) \frac{\partial u}{\partial n} \mathrm{~d} s_{1} .
$$

The capacity of the two-dimensional capacitor with non-homogeneous dielectric material is defined as

$$
C=\frac{Q_{1}}{U_{1}}=\oint_{\partial A_{1}} \varepsilon(\mathbf{r}) \frac{\partial u}{\partial n} \mathrm{~d} s_{1} .
$$

From Eq. (19) and Eq. (20) we obtain

$$
C=\int_{A} \varepsilon(\mathbf{r})|\nabla u|^{2} \mathrm{~d} A .
$$

\section{Bounds for the capacity}

\subsection{Upper bound}

Theorem 1. If any function $F=F(\mathbf{r})$ which is continuously differentiable in the plane domain $\bar{A}=$ $A \cup \partial A$ satisfies the boundary conditions

$$
F(\mathbf{r})=1, \quad \mathbf{r} \in \partial A_{1}, \quad F(\mathbf{r})=0, \quad \mathbf{r} \in \partial A_{2},
$$

then the inequality relatoin

$$
C \leq \int_{A} \varepsilon(\mathbf{r})|\nabla F|^{2} \mathrm{~d} A
$$

is true.

Proof. The proof of upper bound formula for $C$ is based on the following Cauchy-Schwarz inequality relation

$$
\begin{gathered}
\left(\int_{A} \varepsilon(\mathbf{r}) \nabla F \cdot \nabla u \mathrm{~d} A\right)^{2} \leq \\
\leq \int_{A} \varepsilon(\mathbf{r})|\nabla F|^{2} \mathrm{~d} A \int_{A} \varepsilon(\mathbf{r})|\nabla u|^{2} \mathrm{~d} A .
\end{gathered}
$$

A simple computation gives

$$
\begin{gathered}
\int_{A} \varepsilon(\mathbf{r}) \nabla F \cdot \nabla u \mathrm{~d} A= \\
=\int_{A} \nabla \cdot(F \varepsilon(\mathbf{r}) \nabla u) \mathrm{d} A-\int_{A} F \nabla \cdot(\varepsilon(\mathbf{r}) \nabla u) \mathrm{d} A= \\
=\oint_{\partial A_{1}} \varepsilon(\mathbf{r}) \frac{\partial u}{\partial n} \mathrm{~d} s=\int_{A} \varepsilon(\mathbf{r})|\nabla u|^{2} \mathrm{~d} A
\end{gathered}
$$

according to Eqs. (20) and (21). The combination of the inequality relation (24) with Eq. (25) gives the upper bound formula (23). A brief investigation based on Cauchy-Schwarz inequality (24) shows that equality in relation (23) is valid only if $F(\mathbf{r}) \equiv u(\mathbf{r})$.

\subsection{Lower bound}

Theorem 2. Let $\mathbf{q}=\mathbf{q}(\mathbf{r})$ be a two-dimensional vector field defined in the plane domain $\bar{A}=A \cup \partial A$. The identically non-zero vector field $\mathbf{q}=\mathbf{q}(\mathbf{r})$ satisfies the following equation

$$
\nabla \cdot(\varepsilon(\mathbf{r}) \mathbf{q})=0, \quad \mathbf{r} \in A .
$$


In this case we have

$$
C \geq \frac{\left(\oint_{\partial A_{1}} \varepsilon(\mathbf{r}) \mathbf{q} \cdot \mathbf{n} \mathrm{d} s_{1}\right)^{2}}{\int_{A} \varepsilon(\mathbf{r}) \mathbf{q}^{2} \mathrm{~d} A} .
$$

Proof. The validity of the lower bound formula (27) follows from the following Cauchy-Schwarz inequality

$$
\begin{gathered}
\left(\int_{A} \varepsilon(\mathbf{r}) \mathbf{q} \cdot \nabla u \mathrm{~d} A\right)^{2} \leq \\
\leq \int_{A} \varepsilon(\mathbf{r}) \mathbf{q}^{2} \mathrm{~d} A \int_{A} \varepsilon(\mathbf{r})|\nabla u|^{2} \mathrm{~d} A .
\end{gathered}
$$

A simple computation yields the result

$$
\begin{aligned}
& \int_{A} \varepsilon(\mathbf{r}) \mathbf{q} \cdot \nabla u \mathrm{~d} A=\int_{A} \nabla \cdot(u \varepsilon(\mathbf{r}) \mathbf{q}) \mathrm{d} A- \\
& -\int_{A} u \nabla \cdot(\varepsilon(\mathbf{r}) \mathbf{q}) \mathrm{d} A=\oint_{\partial A_{1}} \varepsilon(\mathbf{r}) \mathbf{q} \cdot \mathbf{n} \mathrm{d} s_{1} .
\end{aligned}
$$

Substitution Eq. (29) into the inequality relation (28) provides

$$
C=\int_{A} \varepsilon(\mathbf{r})|\nabla u|^{2} \mathrm{~d} A \geq \frac{\left(\underset{\partial A_{1}}{\oint_{A}} \varepsilon(\mathbf{r}) \mathbf{q} \cdot \mathbf{n} \mathrm{d} s_{1}\right)^{2}}{\int_{A} \varepsilon(\mathbf{r}) \mathbf{q}^{2} \mathrm{~d} A},
$$

which is the lower bound formula (27). Equality in (27) is reached only if

$$
\mathbf{q}=\lambda \nabla u, \quad \mathbf{r} \in A \cup \partial A,
$$

where $\lambda$ is a real number which is different from zero, otherwise, it is an arbitrary real number. The validity of this statement follows from Eq. (19).

Theorem 3. Let $f=f(\mathbf{r})$ be non-identically constant function in $\bar{A}=A \cup \partial A$ which satisfies the Laplace equation in $A$

$$
\triangle f=0, \quad \mathbf{r} \in A
$$

The following lower bound for the capacitance $C$ is valid

$$
C \geq \frac{\left(\oint_{\partial A_{1}} \frac{\partial f}{\partial n} \mathrm{~d} s_{1}\right)^{2}}{\int_{A} \frac{|\nabla f|^{2}}{\varepsilon(\mathbf{r})} \mathrm{d} A} .
$$

Proof. The proof of the lower bound (33) can be obtained from inequality relation (30) by the following substitution

$$
\mathbf{q}=\frac{\nabla f}{\varepsilon(\mathbf{r})}, \quad \mathbf{r} \in A \cup \partial A .
$$

\section{Numerical examples}

\subsection{Example}

Let

$$
r=R_{i}(\varphi), \quad 0 \leq \varphi \leq 2 \pi, \quad(i=1,2)
$$

be the equation of the boundary curve $\partial A_{i}(i=1,2)$ which is shown in Fig. 2. To obtain the upper bound for the capacitance we can use the following functions which satisfy the boundary condition (22)

$$
\begin{gathered}
F(r, \varphi)=\frac{\ln \frac{R_{2}(\varphi)}{r}}{\ln \frac{R_{2}(\varphi)}{R_{1}(\varphi)}}, \\
F(r, \varphi)=\frac{r-R_{2}(\varphi)}{R_{1}(\varphi)-R_{2}(\varphi)} .
\end{gathered}
$$

In order we can use the lower bound formula (33) we consider the next harmonic function

$$
f(r, \varphi)=\ln r .
$$

For this function we have

$$
\begin{gathered}
|\nabla f|^{2}=\frac{1}{r^{2}}, \\
\frac{\partial f}{\partial n}=\nabla f \cdot \mathbf{n}=\frac{1}{r} \mathbf{e}_{r} \cdot \mathbf{n} .
\end{gathered}
$$

On the boundary curve $\partial A_{1}$

$$
\begin{gathered}
\mathbf{n}=\frac{R_{1}(\varphi) \mathbf{e}_{r}-\frac{\mathrm{d} R_{1}}{\mathrm{~d} \varphi} \mathbf{e}_{\varphi}}{\sqrt{R_{1}^{2}(\varphi)+\left(\frac{\mathrm{d} R_{1}}{\mathrm{~d} \varphi}\right)^{2}},} \\
\mathrm{~d} s=\sqrt{R_{1}^{2}(\varphi)+\left(\frac{\mathrm{d} R_{1}}{\mathrm{~d} \varphi}\right)^{2}} \mathrm{~d} \varphi, \\
\oint_{\partial A_{1}} \frac{\partial f}{\partial n} \mathrm{~d} s=2 \pi, \\
\int_{A} \frac{|\nabla f|^{2}}{\varepsilon(r)} \mathrm{d} A=\int_{0}^{2 \pi} \int_{R_{1}(\varphi)}^{R_{2}(\varphi)} \frac{\mathrm{d} r}{r \varepsilon(r)} \mathrm{d} \varphi .
\end{gathered}
$$

The lower bound formula for the function $(38)$ has the following form

$$
C \geq \frac{4 \pi^{2}}{\int_{0}^{2 \pi} \int_{R_{1}(\varphi)}^{R_{2}(\varphi)} \frac{\mathrm{d} r}{r \varepsilon(r)} \mathrm{d} \varphi}
$$




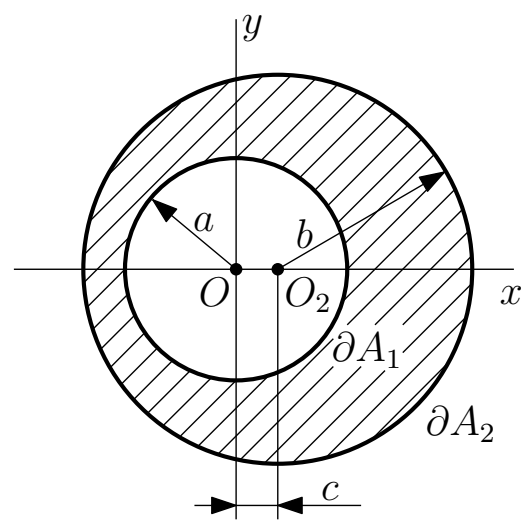

Figure 3: A plane domain bounded by eccentrically located circles.

\subsection{Example}

The formulae of Example 4.1 will be used to estimate capacitance of the two-dimensional capacitor shown in Fig. 3. The boundary curves are circles whose centres do not coincide (Fig. 3). The following data are used in the numerical computation: $a=0.018 \mathrm{~m}$, $b=0.03 \mathrm{~m} c=0.0025 \mathrm{~m}, \varepsilon_{0}=8.856 \times 10^{-12}$ $\mathrm{C} / \mathrm{mV}, k=3.5, \varepsilon(r)=k \varepsilon_{0} r$. In this example

$$
\begin{gathered}
R_{1}(\varphi)=a, \\
R_{2}(\varphi)=c \cos \varphi+\sqrt{c^{2} \cos ^{2} \varphi+b^{2}-c^{2}} .
\end{gathered}
$$

Application of bounding formula (23) for the function (36) gives

$$
C \leq 9.108854951 \times 10^{-12} \mathrm{~F} .
$$

In the present case from the lower bound formula (45) we obtain

$$
C \geq 8.83375633 \times 10^{-12} \mathrm{~F} .
$$

For homogeneous capacitor when $\varepsilon(\mathbf{r})=\varepsilon_{0}$ the above used bounding formulae provide the following results assuming that $a=0.018 \mathrm{~m}, b=0.03 \mathrm{~m}$, $c=0.0025 \mathrm{~m}$

$$
1.1001154 \times 10^{-10} \mathrm{~F}<C<1.1484411 \times 10^{-10} \mathrm{~F} \text {. }
$$

The exact value of the capacitance in this case [5]

$$
C=1.131134773 \times 10^{-10} \mathrm{~F} .
$$

\subsection{Example}

In this example we consider a plane capacitor whose boundary curves are confocal ellipses. To develop the estimation formulae it is necessary to introduce an orthogonal curvilinear coordinate system. The definition of the curvilinear coordinates $\rho, \alpha$ is given by the

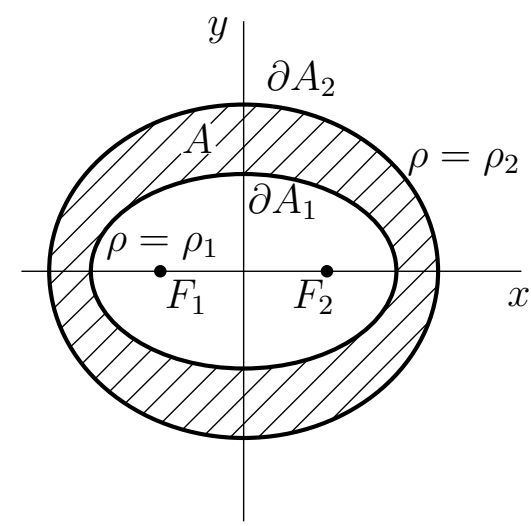

Figure 4: Capacitor bounded by two confocal ellipses.

following equations

$$
\begin{gathered}
x=\left(\rho+\frac{c^{2}}{4 \rho}\right) \cos \varphi, \quad y=\left(\rho-\frac{c^{2}}{4 \rho}\right) \sin \varphi, \\
\rho_{1} \leq \rho \leq \rho_{2}, \quad 0 \leq \varphi \leq 2 \pi .
\end{gathered}
$$

The capacitor with confocal elliptical boundary curves is shown in Fig. 4. From Eq. (51) it follows that the semi axes of the boundary ellipses are

$$
\begin{gathered}
a_{1}=\sqrt{\rho_{1}^{2}+\frac{c^{2}}{2}+\frac{c^{4}}{16 \rho_{1}^{2}}}, \\
b_{1}=\sqrt{\rho_{1}^{2}-\frac{c^{2}}{2}+\frac{c^{4}}{\rho_{1}^{2}}}, \\
a_{2}=\sqrt{\rho_{2}^{2}+\frac{c^{2}}{2}+\frac{c^{4}}{16 \rho_{2}^{2}}}, \\
b_{2}=\sqrt{\rho_{2}^{2}-\frac{c^{2}}{2}+\frac{c^{4}}{\rho_{2}^{2}}},
\end{gathered}
$$

It is evident (Fig. 4)

$$
a_{1}^{2}-b_{1}^{2}=a_{2}^{2}-b_{2}^{2}=c^{2} .
$$

To obtain a lower bound for the capacitance we use next function

$$
F=F(\rho), \quad \rho_{1} \leq \rho \leq \rho_{2}, \quad 0 \leq \varphi \leq 2 \pi .
$$

The test function $F=F(\rho)$ satisfies the boundary conditions

$$
F\left(\rho_{1}\right)=1, \quad F\left(\rho_{2}\right)=0 .
$$

Substitution of Eq. (55) into the upper bound formula (23) yields

$$
C \leq \int_{A} \varepsilon(\rho)|\nabla F|^{2} \mathrm{~d} A
$$


assuming that $\varepsilon$ depends only on the curvilinear coordinates $\rho$. The area element in the $(\rho, \alpha)$ curvilinear coordinate system is as follows

$$
\mathrm{d} A=H_{\rho} H_{\alpha} \mathrm{d} \rho \mathrm{d} \alpha,
$$

where $H_{\rho}$ and $H_{\alpha}$ are the Lamé coefficients

$$
\begin{gathered}
H_{\rho}^{2}=\left(\frac{\partial x}{\partial \rho}\right)^{2}+\left(\frac{\partial y}{\partial \rho}\right)^{2} \\
H_{\alpha}^{2}=\left(\frac{\partial x}{\partial \alpha}\right)^{2}+\left(\frac{\partial y}{\partial \alpha}\right)^{2}=\rho^{2} H_{\rho}^{2} .
\end{gathered}
$$

In the curvilinear coordinates

$$
\nabla F=\frac{1}{H_{\rho}} \frac{\partial F}{\partial \rho} \mathbf{e}_{\rho}+\frac{1}{H_{\alpha}} \frac{\partial F}{\partial \alpha} \mathbf{e}_{\alpha},
$$

where $\mathbf{e}_{\rho}$ and $\mathbf{e}_{\alpha}$ are the unit vectors of the curvilinear coordinate system $(\rho, \alpha)$ that is

$$
\begin{gathered}
\mathbf{e}_{\rho}=\frac{1}{\left|\frac{\partial \mathbf{r}}{\partial \rho}\right|} \frac{\partial \mathbf{r}}{\partial \rho}=\frac{1}{H_{\rho}} \frac{\partial \mathbf{r}}{\partial \rho}, \\
\mathbf{e}_{\alpha}=\frac{1}{\left|\frac{\partial \mathbf{r}}{\partial \alpha}\right|} \frac{\partial \mathbf{r}}{\partial \alpha}=\frac{1}{H_{\alpha}} \frac{\partial \mathbf{r}}{\partial \alpha} .
\end{gathered}
$$

In present problem we have

$$
|\nabla F|^{2}=\frac{1}{H_{\rho}^{2}}\left(\frac{\partial F}{\partial \rho}\right)^{2} .
$$

Substitution of Eqs. (58), (63) into the upper bound inequality (57) gives

$$
\begin{aligned}
C & \leq \int_{\alpha=0}^{2 \pi} \int_{\rho=\rho_{1}}^{\rho_{2}} \rho \varepsilon(\rho)\left(\frac{\partial F}{\partial \rho}\right)^{2} \mathrm{~d} \rho \mathrm{d} \alpha= \\
& =2 \pi \int_{\rho_{1}}^{\rho_{2}} \rho \varepsilon(\rho)\left(\frac{\partial F}{\partial \rho}\right)^{2} \mathrm{~d} \rho .
\end{aligned}
$$

By the application of the known results of variational calculus [16, 17] it can be pointed out that the upper bound (64) is the sharpest in the case

$$
F(\rho)=1-\frac{\int_{\rho_{1}}^{\rho} \frac{\mathrm{d} \lambda}{\lambda \varepsilon(\lambda)}}{\int_{\rho_{1}} \frac{\mathrm{d} \rho}{\rho \varepsilon(\rho)}},
$$

The substitution of the expression $F=F(\rho)$ given by Eq. (65) into the upper bound formula (64) gives

$$
C \leq \frac{2 \pi}{\int_{\rho_{1}}^{\rho_{2}} \frac{\mathrm{d} \rho}{\rho \varepsilon(\rho)}} .
$$

It is known the Laplace operator has the form in orthogonal curvilinear coordinate system $(\alpha, \rho)$

$$
\triangle P=\frac{1}{\rho H_{\rho}^{2}}\left[\frac{\partial}{\partial \rho}\left(\rho \frac{\partial P}{\partial \rho}\right)+\frac{\partial}{\partial \alpha}\left(\frac{1}{\rho} \frac{\partial P}{\partial \alpha}\right)\right],
$$

where $P=P(\alpha, \rho)$ is a given function of the curvilinear coordinates $\alpha$ and $\rho$. Using Eq. (67) it can be shown that

$$
f(\rho)=\ln \rho, \quad 0<\rho_{1} \leq \rho \leq \rho_{2}
$$

is a regular harmonic function in the doubly connected plane domain $A$. Our aim is to give a lower bound for the capacitance by the use of lower bound formula (33). A simple computation gives

$$
|\nabla f|^{2}=\left(\frac{1}{\rho H_{\rho}}\right)^{2}
$$

$$
\begin{aligned}
\int_{A} \frac{|\nabla f|^{2}}{\varepsilon(\rho)} \mathrm{d} A= & \int_{\alpha=0}^{2 \pi} \int_{\rho=\rho_{1}}^{\rho_{2}} \frac{1}{\rho^{2} H_{\rho}^{2} \varepsilon(\rho)} \rho H_{\rho}^{2} \mathrm{~d} \rho \mathrm{d} \alpha= \\
& =2 \pi \int_{\rho_{1}}^{\rho_{2}} \frac{\mathrm{d} \rho}{\rho \varepsilon(\rho)},
\end{aligned}
$$

$$
\begin{gathered}
\left(\frac{\partial f}{\partial n}\right)_{\rho_{1}}=\left(\frac{1}{H_{\rho}} \frac{1}{\rho}\right)_{\rho=\rho_{1}}, \\
\mathrm{~d} s_{1}=H_{\alpha} \mathrm{d} \alpha=\left(\rho H_{\rho}\right)_{\rho=\rho_{1}} \mathrm{~d} \alpha .
\end{gathered}
$$

From Eqs. (71) and (72) we obtain

$$
\oint_{\partial A_{1}} \frac{\partial f}{\partial n} \mathrm{~d} s_{1}=2 \pi .
$$

Substitution of equation (70), (73) into the lower bound formula (33) yields the result

$$
C \geq \frac{2 \pi}{\int_{\rho_{1}}^{\rho_{2}} \frac{\mathrm{d} \rho}{\rho \varepsilon(\rho)}} .
$$

It follows from the equality of the upper bound (66) and lower bound (74) that the exact value of the capacitance of the two-dimensional capacitor in the present problem is

$$
C=\frac{2 \pi}{\int_{\rho_{1}}^{\rho_{2}} \frac{\mathrm{d} \rho}{\rho \varepsilon(\rho)}} .
$$




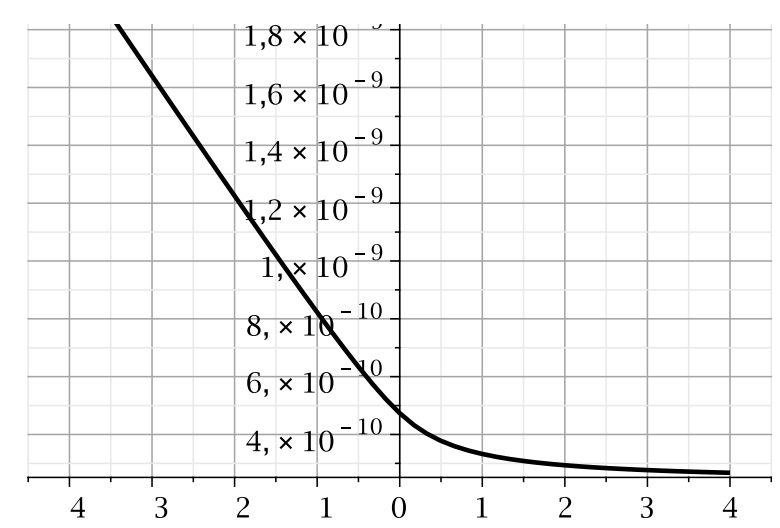

Figure 5: The graph of the capacitance as a function of power index $n$ for $-4 \leq n \leq 4$.

We illustrate the application of formula $(75)$ for functionally graded material when the permittivity of the non-homogeneous dielectric material is power law graded as function of $\rho$

$$
\varepsilon(\rho, \alpha)=k_{1} \varepsilon_{0}+\varepsilon_{0}\left(k_{2}-k_{1}\right)\left(\frac{\rho-\rho_{1}}{\rho_{2}-\rho_{1}}\right)^{n} .
$$

In Eq. (76), $k_{1}$ and $k_{2}$ are the relative permittivity of the components material of the composite dielectric material, $n$ is the power index. The following data will be used in the numerical example: $\rho_{1}=0.5 \mathrm{~m}$, $\rho_{2}=0.8 \mathrm{~m}, c=0.25 \mathrm{~m}, \varepsilon_{0}=8.856 \times 10^{-12} \mathrm{~F} / \mathrm{m}$, $k_{1}=2, k_{2}=4$. Fig. 5 illustrates the capacitance as a function of the power index $n$ for $-4 \leq n \leq 4$.

\section{Conclusions}

Upper and lower bounds for the capacitance of twodimensional capacitor are proven by the application of Cauchy-Schwarz inequality. The considered cylindrical capacitor consists of non-homogeneous dielectric materials. The derived upper and lower bound formulae of capacitance can be used to check the results of numerical computations obtained by finite element method, boundary element method and by other numerical methods.

\section{References:}

[1] J. D Jackson, Classical electrodynamics, Wiley and Sons, New York, 1988.

[2] V. V. Batygin, I. N. Toptygin, Problems in Electrodynamics, Academic Press, New York, 1965.

[3] L. Solymar, Lectures on Electromagnetic Theory: a Short Course for Engineers, Oxford University Press, Oxford, 1984.

[4] L. D. Landau, E. M. Lifshitz, Electrodynamics of Continuous Media. Pergamon Press, Oxford, 1963.

[5] K. Simonyi, Foundations of Electrical Engineering: Fields-Networks-Waves, Pergamon Press, Oxford, 1963.
[6] E. W. Bai, K. E. Lonngren, The spherical capacitor: a vehicle to introduce numerical methods, Computers and Electrical Engineering, Vol.29, No.1, 2003, pp. 231-234.

[7] D. F. Bartlett, T. R. Corle, The circular parallel plate capacitor: a numerical solution for the potential, Journal of Physics A: Mathematical and General, Vol.18, No.9, 1985, pp. 1337.

[8] G. Paffuti, E. Cataldo, A. Di Lieto, F. Maccarrone, Circular plate capacitor with different discs, Proceedings of the Royal Society A Mathematical, Physical and Engineering Sciences, Vol.472, No.2194, 2016, 20160574.

[9] W. E. Parr, Upper and Lower Bounds for the Capacitance of the Regular Solids Journal of the Society for Industrial and Applied Mathematics, Vol.9, No.3, 1961, pp. 334-386.

[10] D. W. Kammler, An Error Bound for Capacitance Calculations, SIAM Journal on Numerical Analysis, Vol.6, No.2, 1969, pp. 254-257.

[11] H. J. Wintle, The capacitance of the cube and square plate by random walk methods, Journal of Electrostatics, Vol.62, No.1, 2004, pp. 51-62.

[12] T. V. Rao, Capacity of the circular plate condenser: analytical solutions for large gaps between the plates, Journal of Physics A: Mathematical and General, Vol.38, No.46, 2005, 10037.

[13] G. J. Sloggett, N. G. Barton, S. J. Spencer, Fringing fields in disc capacitors, Journal of Physics A: Mathematical and General, Vol.19, No.14, 1986, 2725.

[14] M. Nithin, H. M. Kittur, Design of On Chip Spiral Inductors for Millimeter Wave Frequency Synthesizers, International Journal of Circuits, Systems and Signal Processing, Vol. 14, 2020, pp. 191-196.

[15] G. P. Arada, How Varying the Dipole Lengths of a Uniform Linear Array Affects the Performance of an ESPRIT-based Direction Finding Algorithm, International Journal of Circuits, Systems and Signal Processing, Vol. 14, 2020, pp. 952-958.

[16] R. Weinstock, Calculus of Variations, McGrawHill, New York, 1952.

[17] P. K. Chattopadhyay, Mathematical Physics, New Age International Ltd. Publishers, New Delhi, 2004.

\section{Creative Commons Attribution License 4.0} (Attribution 4.0 International, CC BY 4.0)

This article is published under the terms of the Creative Commons Attribution License 4.0

https://creativecommons.org/licenses/by/4.0/deed.en_US 